\title{
Lung Epithelial Cell-Specific Expression of Human Lysosomal Acid Lipase Ameliorates Lung Inflammation and Tumor Metastasis in Lipa ${ }^{-/-}$Mice
}

\author{
Ting Zhao, ${ }^{*}$ Xinchun Ding, ${ }^{*}$ Hong $\mathrm{Du}^{* \dagger}$ and Cong Yan*${ }^{* \dagger}$
}

From the Department of Pathology and Laboratory Medicine, ${ }^{*}$ and the Indiana University Simon Cancer Center, ${ }^{\dagger}$ Indiana University School of Medicine, Indianapolis, Indiana

\author{
Accepted for publication \\ April 13, 2016. \\ Address correspondence to \\ Cong Yan, Ph.D., Department \\ of Pathology and Laboratory \\ Medicine, Indiana University \\ School of Medicine, $975 \mathrm{~W}$ \\ Walnut St, IB424G, Indian- \\ apolis, IN 46202; or Hong Du, \\ Ph.D., Department of Pathology \\ and Laboratory Medicine, \\ Indiana University School of \\ Medicine, 975 W Walnut St, \\ IB424E, Indianapolis, IN \\ 46202. \\ E-mail: coyan@iupui.edu or \\ hongdu@iupui.edu.
}

\begin{abstract}
Lysosomal acid lipase (LAL), a key enzyme in the metabolic pathway of neutral lipids, has a close connection with inflammation and tumor progression. One major manifestation in LAL-deficient $\left(\mathrm{Lipa}^{-{ }^{-}}\right)$ mice is an increase of tumor growth and metastasis associated with expansion of myeloid-derived suppressor cells. In the lung, LAL is highly expressed in alveolar type II epithelial cells. To assess how LAL in lung epithelial cells plays a role in this inflammation-related pathogenic process, lung alveolar type II epithelial cell-specific expression of human $\mathrm{LAL}$ (hLAL) in $\mathrm{Lipa}^{-/-}$mice was established by crossbreeding of CCSP-driven rtTA transgene and (Tet0) $)_{7}$-CMV-hLAL transgene into Lipa $^{-1-}$ mice (CCSP-Tg/KO). hLAL expression in lung epithelial cells not only reduced tumor-promoting myeloid-derived suppressor cells in the lung, but also down-regulated the synthesis and secretion of tumor-promoting cytokines and chemokines into the bronchoalveolar lavage fluid of $\mathrm{Lipa}^{-1-}$ mice. $\mathrm{hLAL}$ expression reduced the immunosuppressive functions of bronchoalveolar lavage fluid cells, inhibited bone marrow cell transendothelial migration, and inhibited endothelial cell proliferation and migration in Lipa $^{-/-}$ mice. As a result, hLAL expression in CCSP-Tg/KO mice corrected pulmonary damage, and inhibited tumor cell proliferation and migration in vitro, and tumor metastasis to the lung in vivo. These results support a concept that LAL is a critical metabolic enzyme in lung epithelial cells that regulates lung homeostasis, immune response, and tumor metastasis. (Am J Pathol 2016, 186: 2183-2192; http:// dx.doi.org/10.1016/j.ajpath.2016.04.014)
\end{abstract}

The lung not only provides an interface for gas exchange between the air and blood to supply oxygen and remove carbon dioxide, but also actively participates in inflammatory responses against pathogens and various diseases. ${ }^{1}$ The lung is a highly lipophilic organ and covered with pulmonary surfactant. The round secretory alveolar type II (AT II) epithelial cells scattered around alveolar type I epithelial cells, usually in the corner of the alveoli, are a critical cell type involved in many lung functions. AT II epithelial cells serve as local stem cells to differentiate into AT I epithelial cells during repair of lung injury. They synthesize and secrete surfactant through lamellar bodies rich in phospholipids, which spread out to form a membrane on the surface of the interalveolar walls to prevent the lung from collapsing during respiratory cycles. AT II epithelial cells are able to transform into cancer cells when triggered by overexpression of oncogenic molecules that are downstream genes or effectors of lysosomal acid lipase (LAL; eg, Stat3, MMP12, and Api6). ${ }^{2-5}$ In a separate function, AT II epithelial cells serve as local immune cells, which secrete proinflammatory cytokines and chemokines to actively participate in the regional pulmonary microenvironment to form niches for tumor growth and metastasis in the lung.

LAL, hydrolyzing cholesteryl esters, and triglycerides in the lysosome of cells to generate free fatty acids and cholesterol, is a key enzyme in the metabolic pathway of neutral lipids. The close connection between LAL and inflammation has been

Supported by NIH grants CA138759 (C.Y.), CA152099 (C.Y.), and HL087001 (H.D.).

Disclosures: The authors have submitted a provisional patent on lysosomal acid lipase as an immune therapy for cancer treatment. 
well documented. ${ }^{6-9}$ Genetic ablation of the Lipa gene in mice has resulted in pulmonary inflammation. The high inflammatory state causes remodeling of the alveolar structure, pulmonary emphysema, and Clara cell hypertrophy and hyperplasia. ${ }^{6}$ One of the most important manifestations associated with these pathogeneses is the increased proinflammatory cytokines/chemokines and infiltration of myeloid-derived suppressor cells (MDSCs) into the lung. ${ }^{7,10}$ MDSCs possess dual functions to facilitate cancer metastasis in the lung: suppression of immune surveillance and direct stimulation of cancer cell proliferation and growth. ${ }^{11}$

To better understand the physiological and pathological role of LAL in lung epithelial cells, AT II epithelial cell-specific expression of human LAL (hLAL) in $\mathrm{Lipa}^{-/-}$ mice was established by crossbreeding previously generated CCSP-rtTA transgenic mice ${ }^{12}$ and (TetO) $)_{7}$-CMV-hLAL transgenic mice ${ }^{13}$ into Lipa $^{-1-}$ mice (named CCSP-Tg/KO mice). The results demonstrated that LAL in lung epithelial cells plays an important role in controlling lung proinflammatory cytokine/chemokine production, infiltration of MDSCs into the lung, and tumor metastasis.

\section{Materials and Methods}

\section{Animals and Cell Lines}

Wild-type $\left(\mathrm{Lipa}^{+/+}\right.$; referred here on as $\mathrm{Lal}^{+/+}$) and $\mathrm{Lipa}^{-/-}$ (referred here on as $\mathrm{Lal}^{-1-}$ ) mice of the FVB/N background were bred in house. CCSP-rtTA/(TetO) ${ }_{7}$-CMV-hLAL; $\mathrm{Lal}^{-/-}$ (CCSP-Tg/KO) triple mice were generated by crossbreeding previously generated CCSP-rtTA transgenic mice ${ }^{12}$ and $(\text { TetO })_{7}$-CMV-hLAL transgenic mice ${ }^{13}$ into Lal $^{-1-}$ mice. This triple transgenic mouse model is lung AT II epithelial cellspecific Tet-on expression of wild-type hLAL in $\mathrm{Lal}^{-1-}$ mice under the control of the CCSP (SCGB1A1) promoter. Mice were used at 4 months of age, and they had been treated with or without doxycycline for 3 months. All scientific protocols involving the use of animals have been approved by the Institutional Animal Care and Use Committee of Indiana University School of Medicine and followed guidelines established by the Panel on Euthanasia of the American Veterinary Medical Association. Animals were housed under Institutional Animal Care and Use Committee-approved conditions in a secured animal facility at Indiana University School of Medicine.

The murine B16 melanoma cell line and Lewis lung carcinoma (LLC) cell line (ATCC, Manassas, VA) were cultured in Dulbecco's modified Eagle's medium (DMEM) supplemented with $10 \%$ fetal bovine serum (FBS; Gibco, Grand Island, NY).

\section{Flow Cytometry Analysis}

For immune cell profile analysis, single cells from the lung, bone marrow, spleen, and blood of $\mathrm{Lal}^{+/+}, \mathrm{Lal}^{-/-}$, and $\mathrm{CCSP}-\mathrm{Tg} / \mathrm{KO}$ triple mice were prepared as previously described. ${ }^{14,15}$ Cells were labeled with isotype control or surface-specific primary antibodies at $4{ }^{\circ} \mathrm{C}$ for 15 minutes, and then washed and prepared for flow cytometry analysis. Anti-CD11b (M1/70) PE-Cyanine7 and anti-Ly6G (RB68c5) APC-eFluor 780 were purchased from eBiosciences (San Diego, CA). For characterization of tissue-specific expression of the hLAL transgene, single cells from the lungs of $\mathrm{Lal}^{+/+}, \mathrm{Lal}^{-/-}$, and CCSP-Tg/KO triple mice were prepared and stained as previously described. ${ }^{4}$ Surfactant protein C (AT II epithelial cell marker) Ab and Flag Ab were purchased from Santa Cruz Biotechnology (Dallas, TX) and Sigma-Aldrich (St. Louis, MO), respectively. In gated surfactant protein $\mathrm{C}$-positive cells, the number of the $\mathrm{Flag}^{+}$ cells was analyzed by flow cytometry and calculated on the basis of M1 that is defined by isotype control. Whole spleen cells were used as a control, in which the number of the Flag ${ }^{+}$ cells was analyzed. For flow cytometry analysis, $\geq 30,000$ cells were acquired and scored using a LSRII machine (BD Biosciences, San Jose, CA). Data were processed using the CellQuest software version 5.2.1 (BD Biosciences).

\section{Histology and Immunohistochemical Staining}

The lungs were harvested and fixed with $4 \%$ paraformaldehyde in phosphate-buffered saline (PBS) at $4^{\circ} \mathrm{C}$ overnight. After embedding in paraffin, tissue sections were cut to $5 \mu \mathrm{m}$ thickness. Hematoxylin and eosin staining was performed by the Histological Core Facility, Department of Pathology and Laboratory Medicine, Indiana University. Images were taken by Nikon microscopy image system (Nikon, Tokyo, Japan). Morphometric analysis was performed on sections taken throughout various lobes of the lungs. Images were analyzed by Nikon NIS Elements imaging software version 4.00.08. A threshold was applied to the spaces of the alveoli. Large vessels, smaller arterioles, and venules were excluded from the study. The alveoli were assessed by area, diameter, and perimeter using the integrated morphometry analysis tool. All data were exported to Microsoft Excel version 2010 (Microsoft, Redmond, WA).

\section{Tumor Colonization Model}

The tumor colonization model has been described recently. ${ }^{11}$ Briefly, $5 \times 10^{5}$ B16 melanoma cells in $200 \mu \mathrm{L}$ PBS were injected into the mice via tail vein. Two weeks after the injection, the mice were sacrificed and the lungs were harvested for examination of tumor cell colonization.

\section{Kwik-Diff Staining of BALF Cells}

Kwik-Diff staining of bronchoalveolar lavage fluid (BALF) cells was performed as we previously described. ${ }^{7}$ BALF was collected by $1 \mathrm{~mL}$ PBS. Cells were then centrifuged and resuspended with $0.5 \mathrm{~mL}$ PBS. Same volume of cell suspension $(125 \mu \mathrm{L})$ was cytospun on the slide and stained with the Kwik-Diff stain kit according to the manufacturer's 
instruction (Thermo Shandon, Pittsburgh, PA). Images were taken by Nikon microscopy image system.

\section{Cytokine Measurement by Enzyme-Linked Immunosorbent Assay}

BALF was collected by $1 \mathrm{~mL}$ PBS. The expression levels of IL-6, granulocyte-macrophage colony-stimulating factor (GM-CSF), chemokine ligand (CCL) 2, and tumor necrosis factor (TNF)- $\alpha$ in the BALF and plasma were measured using enzyme-linked immunosorbent assay kits (BD Biosciences, San Jose, CA), according to the manufacturer's instructions.

\section{Quantitative Real-Time RT-PCR}

Total RNAs were purified from lungs of $\mathrm{Lal}^{+/+}, \mathrm{Lal}^{-/-}$, and CCSP-Tg/KO triple mice using RNeasy Mini Kits, according to the manufacturer's instruction (Qiagen, Valencia, CA). Quantitative RT-PCR was performed as described previously. ${ }^{16}$ Analysis was performed by the $2^{-\Delta \Delta C T}$ method. Primers of mIL-6, mIL-1 $\beta$, mIL-10, mGM-CSF, mM-CSF, mTNF- $\alpha$, mCCL2, mCCL5, and GAPDH for real-time PCR were described previously. ${ }^{17}$

\section{Isolation and in Vitro Culture of Pulmonary ECs}

Endothelial cells (ECs) were isolated from $\mathrm{Lal}^{+/+}$lungs and cultured in vitro as we previously described. ${ }^{18}$ Briefly, the mouse was anesthetized and $1 \mathrm{~mL}$ collagenase A $(2 \mathrm{mg} / \mathrm{mL}$; Roche, Indianapolis, IN) was infused into the lung through the trachea. The lung was removed and then incubated with $10 \mathrm{~mL}$ collagenase A at $37^{\circ} \mathrm{C}$ for 30 minutes. After the incubation, the resulting cell suspension was filtered through a $40 \mu \mathrm{m}$ strainer and centrifuged for 5 minutes at $160 \times g$. After removal of the supernatant, the cell pellet was subjected to magnetic bead sorting using anti-CD31 microbeads (Miltenyi Biotec, Auburn, CA), according to the manufacturer's protocol. The resulting cells were plated onto gelatincoated (Sigma-Aldrich) 6-well plates and maintained in DMEM supplemented with endothelial cell growth supplement, heparin, L-glutamine (Sigma-Aldrich), FBS, and antibiotic-antimycotic (Gibco).

\section{In Vitro BALF Treatment}

BALF was harvested by $1 \mathrm{~mL}$ PBS, and cells were removed by centrifugation. To determine the effect of BALF on tumor cell proliferation, B16 melanoma or LLC cells $(5 \times$ $10^{3}$ ) were seeded into a well of 96-well plate in $100 \mu \mathrm{L}$ DMEM supplemented with 10\% FBS, and then treated with $100 \mu \mathrm{L}$ BALF harvested from $\mathrm{Lal}^{+/+}, \mathrm{Lal}^{-/-}$, or CCSP-Tg/ KO triple mice. Seventy-two hours later, the number of B16 melanoma or LLC cells was counted.

To examine the effect of BALF on EC proliferation, $5 \times$ $10^{4}$ ECs were seeded into a well of 24 -well plates in $250 \mu \mathrm{L}$
DMEM supplemented with $10 \%$ FBS, and then treated with $250 \mu \mathrm{L}$ BALF harvested from $\mathrm{Lal}^{+/+}, \mathrm{Lal}^{-/-}$, or CCSP-Tg/ KO triple mice. Seventy-two hours later, the number of ECs was counted.

To analyze the effects of BALF on tumor cell or EC migration, in vitro wound healing assays were performed as previously described. ${ }^{19}$ Briefly, tumor cells or ECs were seeded at a density of $1.5 \times 10^{5}$ cells/well into a 24 -well plate and incubated overnight to form a confluent monolayer. Scratch was generated by scraping the cell monolayer in a straight line with a p200 pipet tip. After washing three times with PBS, the medium was changed with $250 \mu \mathrm{L}$ DMEM containing $10 \%$ FBS and $5 \mu \mathrm{g} / \mathrm{mL}$ mitomycin C (Sigma-Aldrich). Then, $250 \mu \mathrm{L}$ BALF from $\mathrm{Lal}^{+/+}, \mathrm{Lal}^{-/-}$, or CCSP-Tg/KO triple mice was added to the well. Cells were kept on culture at $37^{\circ} \mathrm{C}, 5 \% \mathrm{CO}_{2}$. Images were taken at 0 and 15 hours after generating the scratch. Migration was estimated by measuring the distances from one side of the scratch to the other side using Nikon NIS Element image software version 4.00.08.

\section{T-Cell Proliferation Assay}

To determine the immunosuppressive effects of BALF cells, BALF was harvested by PBS. After the centrifugation, the resulting cell pellets were collected and used. $\mathrm{CD} 4^{+} \mathrm{T}$ cells were prepared and carboxyfluorescein diacetate succinimyl ester labeled, as we previously described.$^{15}$ Labeled $\mathrm{CD} 4{ }^{+} \mathrm{T}$ cells were co-cultured with BALF cells in 96-well plates precoated with $2 \mu \mathrm{g} / \mathrm{mL}$ anti-CD3 monoclonal antibody (mAb) and $5 \mu \mathrm{g} / \mathrm{mL}$ anti-CD28 $\mathrm{mAb}$ at $37^{\circ} \mathrm{C}, 5 \% \mathrm{CO}_{2}$ for 4 days. The ratio of BALF cells/CD4 ${ }^{+} \mathrm{T}$ cells was $1: 5$. Proliferation of $\mathrm{CD}^{+}{ }^{+} \mathrm{T}$ cells was evaluated as carboxyfluorescein diacetate succinimyl ester dilution by fluorescence activated cell sorting.

\section{Transwell Assay}

Transwell assay was used to determine cell transendothelial migration. ${ }^{18}$ ECs were added to the upper chamber of 24well 8.0- $\mu \mathrm{m}$-pore Transwell plates (Corning, Corning, $\mathrm{NY}$ ), and incubated at $37^{\circ} \mathrm{C}, 5 \% \mathrm{CO}_{2}$ for 48 hours to form an EC monolayer. The supernatant was then removed, and CellTrackerTM Green 5-Chloromethylfluorescein Diacetate (CMFDA) (Invitrogen, Grand Island, NY)-labeled bone marrow cells, MDSCs, or T cells $\left(1 \times 10^{4}\right.$ cells in $200 \mu \mathrm{L}$ media) were added to the upper well. After 4 hours, transendothelial migration of bone marrow cells, MDSCs, or T cells was determined by counting their numbers in the lower chamber under five random microscopic fields.

\section{Statistical Analysis}

Data were expressed as means $\pm \mathrm{SD}$. Differences between two treatment groups were compared by Student's $t$-test. When more than two groups were compared, one-way 
analysis of variance with post hoc Newman-Keul's multiple comparison test was used. Results were considered statistically significant when $P<0.05$. All analyses were performed with GraphPad Prism 5.0 (GraphPad, San Diego, CA).

\section{Results}

\section{hLAL Expression Reduces Lung Destruction}

The specific expression of hLAL protein in lung epithelial cells of doxycycline-treated CCSP-Tg/KO mice was confirmed by flow cytometry analysis. Flag antibody was used to detect hLAL-Flag fusion protein to distinguish from the endogenous murine LAL expression. By flow cytometry analysis, expression of the hLAL-Flag fusion protein was significantly induced in surfactant protein $\mathrm{C}$-positive (AT II cell marker) lung epithelial cells of CCSP-Tg/KO mice with doxycycline treatment (Figure 1A). Consistent with our prediction, no hLAL-Flag fusion protein was detected in the spleen of CCSP-Tg/KO mice regardless of doxycycline treatment (Figure 1A). The transgenic LAL activity under the control of the CCSP promoter has been confirmed before. $^{13}$ As a result, the lung damage observed in $\mathrm{Lal}^{-1-}$ mice was partially restored with hLAL expression in doxycycline-treated CCSP-Tg/KO mice, which was demonstrated by reduced emphysema as assessed by hematoxylin and eosin staining and morphometric measurements of alveolar area, diameter, and perimeter (Figure 1, B and $\mathrm{C}$ ).

\section{hLAL Expression Reduces Cancer Metastasis}

LAL deficiency has been reported to facilitate tumor growth and metastasis. ${ }^{11}$ To evaluate the effects of hLAL expression in lung epithelial cells on tumor metastasis, B16

A
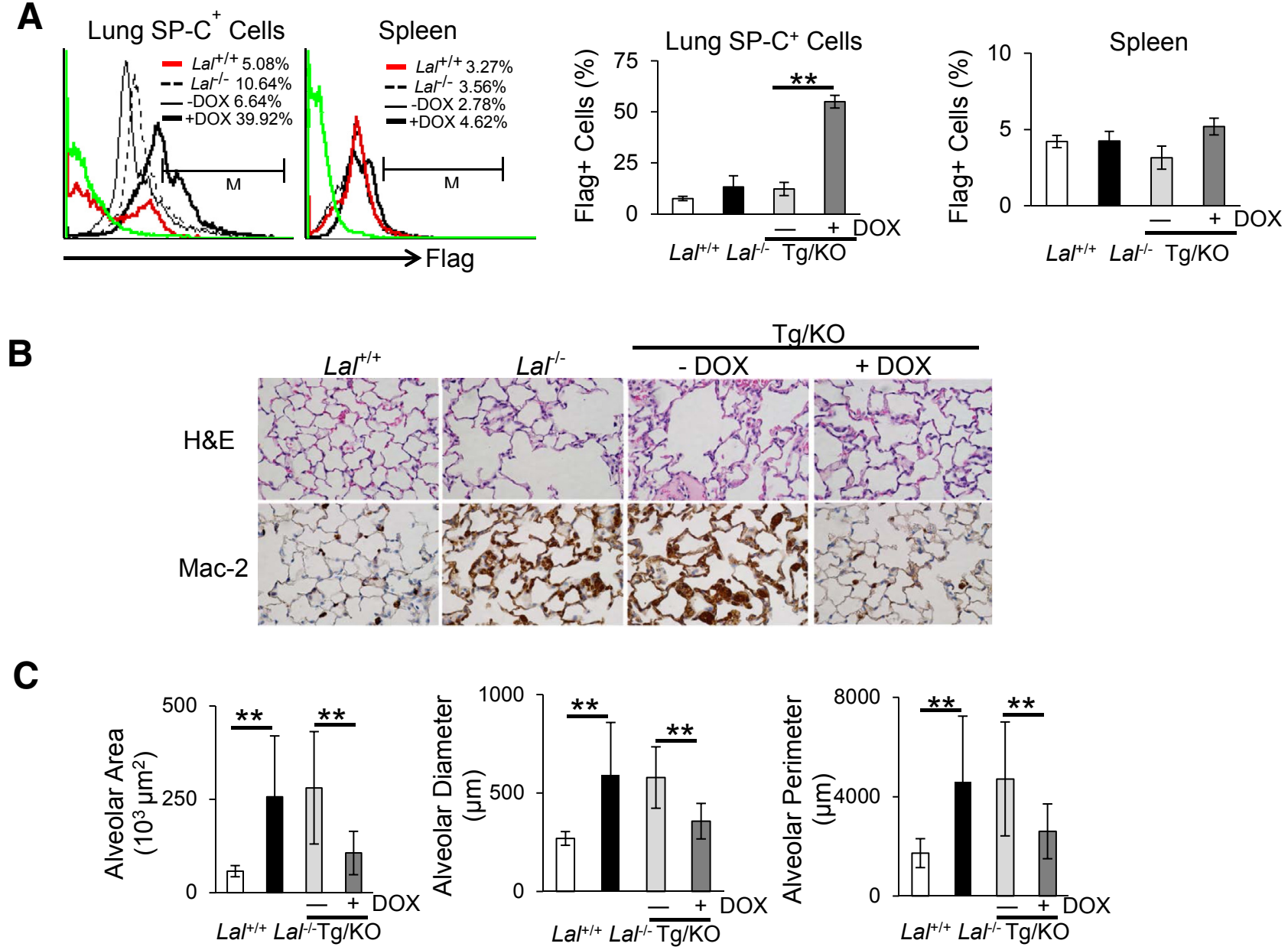

Figure 1 hLAL expression in lung epithelial cells reduces lung destruction in $\mathrm{La}^{-/-}$mice. A: Representative and statistical analyses of hLAL-Flag fusion protein expression in lung surfactant protein $\mathrm{C}-$ positive $\left(\mathrm{SP}-\mathrm{C}^{+}\right)$cells or spleen cells of $\mathrm{Lal}^{+/+}, \mathrm{Lal}^{-/-}$, doxycycline-treated $(+\mathrm{DOX})$, or untreated $(-\mathrm{DOX})$ CCSP-Tg/KO mice by flow cytometry analyses. B: Hematoxylin and eosin (H\&E) and immunohistochemical staining with anti-Mac-2 antibody of lung sections of $\mathrm{Lal}^{+/+}, \mathrm{Lal}^{-/-}$, doxycycline-treated (+DOX), or untreated (-DOX) CCSP-Tg/KO $(\mathrm{Tg} / \mathrm{KO})$ mice. C: Quantitative measurements of alveolar area, diameter, and perimeter in $\mathrm{Lal}^{+/+}, \mathrm{Lal}^{-/-}$, doxycycline-treated, or untreated CCSP-Tg/KO $(\mathrm{Tg} / \mathrm{KO})$ mice were determined by Nikon NIS Elements imaging software version 4.00.08. Data are expressed as means \pm SD (A and C). $n=3$ to 4 (A); $n=10$ (C). ${ }^{* *} P<0.01$. Original magnification, $\times 400$ (B). 
melanoma cells were injected i.v. into CCSP-Tg/KO mice. Two weeks after injection, fewer B16 melanoma colonies were observed in the lungs of doxycycline-treated CCSP$\mathrm{Tg} / \mathrm{KO}$ mice compared to those in untreated mice with statistical significance (Figure 2, A and B). Immunohistochemical staining of lung sections revealed that there were fewer neoplastic melanoma cells and Ki-67-positive proliferative cells in the lungs of doxycycline-treated CCSP-Tg/ $\mathrm{KO}$ mice than those in the lungs of untreated mice (Figure 2C). These observations suggest that lung epithelialspecific expression of hLAL in $\mathrm{Lal}^{-1-}$ mice reduced B16 melanoma cell metastasis.

\section{hLAL Expression Decreases Abnormal Expansion of

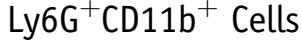

LAL deficiency has been reported to result in severe infiltration and accumulation of tumor-promoting MDSCs in multiple organs of the mice, including the lung. ${ }^{8}$ To test whether lung epithelial expression of hLAL affects the accumulation of $\mathrm{Ly} 6 \mathrm{G}^{+} \mathrm{CD} 11 \mathrm{~b}^{+}$MDSCs, whole cells were harvested from the lungs of doxycycline-treated or untreated $\mathrm{CCSP}-\mathrm{Tg} / \mathrm{KO}$ mice for flow cytometry analysis. Agematched $\mathrm{Lal}^{+/+}$and $\mathrm{Lal}^{-/-}$mice were used as controls. Cells obtained from the bone marrow, blood, and spleen of these mice were also analyzed by flow cytometry analysis. Compared with untreated CCSP-Tg/KO mice, the percentage of $\mathrm{Ly} 6 \mathrm{G}^{+} \mathrm{CD} 11 \mathrm{~b}^{+}$cells in the lung was significantly decreased in doxycycline-treated CCSP-Tg/KO mice; however, there was no reversing effect of $\mathrm{Ly}_{6 \mathrm{G}}{ }^{+} \mathrm{CD} 11 \mathrm{~b}^{+}$cells in the spleen and bone marrow (Figure $3 \mathrm{~A}$ ). $\mathrm{Ly}_{6 \mathrm{G}}{ }^{+} \mathrm{CD} 11 \mathrm{~b}^{+}$ cell accumulation in the blood was reduced with doxycycline treatment as well (Figure 3A), which might be explained by
$\mathrm{hLAL}$, as a secretory enzyme, that was secreted from the lung into circulation. This reduction of $\mathrm{Ly}_{6} \mathrm{G}^{+} \mathrm{CD} 11 \mathrm{~b}^{+}$cells in the lung was confirmed by Kwik-Diff staining of the BALF, in which myeloid cells were significantly reduced in CCSP-Tg/ $\mathrm{KO}$ mice with doxycycline-induced hLAL expression (Figure 3B). Because $>95 \%$ BALF cells are myeloid cells, cells from BALF were harvested to determine their suppressive activity on $\mathrm{T}$ cells. BALF cells from untreated CCSP-Tg/KO mice showed inhibition on T cell proliferation, which was similar to BALF cells from $\mathrm{Lal}^{-1-}$ mice, whereas BALF cells from doxycycline-treated CCSP-Tg/KO mice displayed reduced inhibition on $\mathrm{T}$ cell proliferation (Figure 3C). The above result indicates that the immunosuppressive functions of $\mathrm{Lal}^{-1-}$ BALF cells were impaired by lung epithelial expression of LAL.

\section{hLAL Expression Reduces Synthesis and Secretion of Tumor-Promoting Cytokines and Chemokines}

Cytokines and chemokines play important roles in MDSC recruitment and expansion in lung tumorigenesis,,${ }^{5,20-22}$ and their synthesis and secretion are greatly dependent on the LAL activity. To determine the effects of lung epithelial cell-specific expression of hLAL on the secretion of these cytokines and chemokines, their levels in the BALF were measured by enzyme-linked immunosorbent assay. The IL6, GM-CSF, CCL2, and TNF- $\alpha$ concentrations in BALF were significantly decreased in doxycycline-treated CCSP$\mathrm{Tg} / \mathrm{KO}$ mice compared with those in untreated CCAP-Tg/ KO mice (Figure 4A). On the other hand, the levels of these cytokines and chemokines in the plasma were similar in doxycycline-treated $\mathrm{CCSP}-\mathrm{Tg} / \mathrm{KO}$ mice to those in untreated CCSP-Tg/KO mice (Figure 4B). To confirm this
A

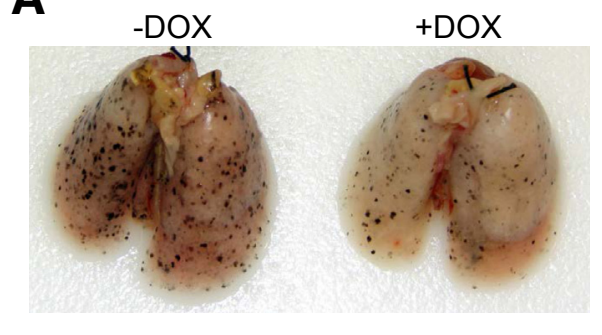

C

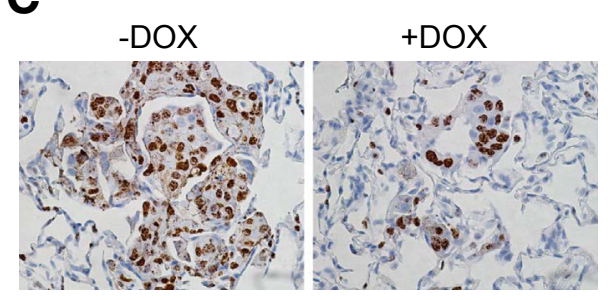

B
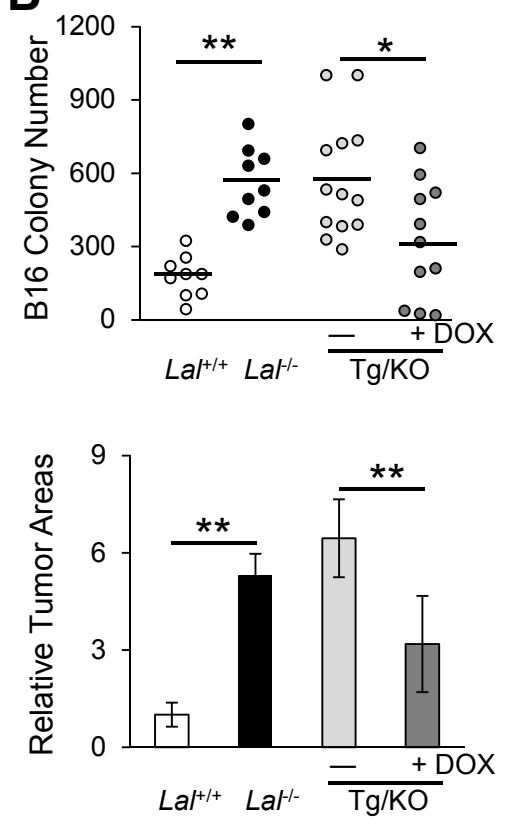

Figure 2 hLAL expression in lung epithelial cells reduces cancer metastasis in $\mathrm{Lal}^{-/-}$mice. A: Representative lungs with metastasized B16 melanoma colonies. B16 melanoma cells $\left(5 \times 10^{5}\right)$ were i.v. injected into doxycycline-treated (+DOX) or untreated (-DOX) CCSP-Tg/KO (Tg/KO) mice for 2 weeks. B: Quantitative analysis of B16 melanoma colonies in the lungs of $\mathrm{Lal}^{+/+}, \mathrm{Lal}^{-/-}$, doxycycline-treated, or untreated CCSP-Tg/KO mice. C: Representative immunohistochemical staining of lung sections from studies in $\mathbf{A}$ using anti-Ki-67 antibody and statistical analysis of relative tumor areas. Data are expressed as means \pm SD (B and C). $n=9$ to 13 (B); $n=10$ (C). ${ }^{*} P<0.05,{ }^{* *} P<0.01$. Original magnification, $\times 400($ C). 

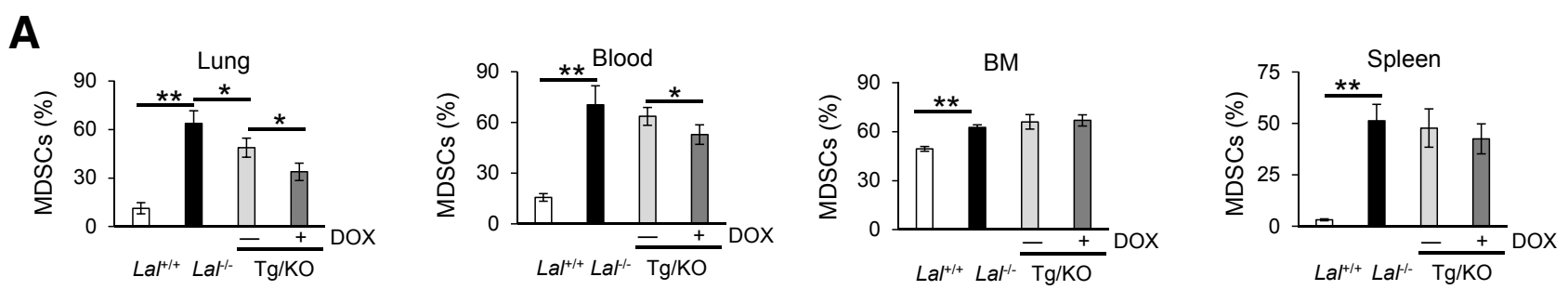

B

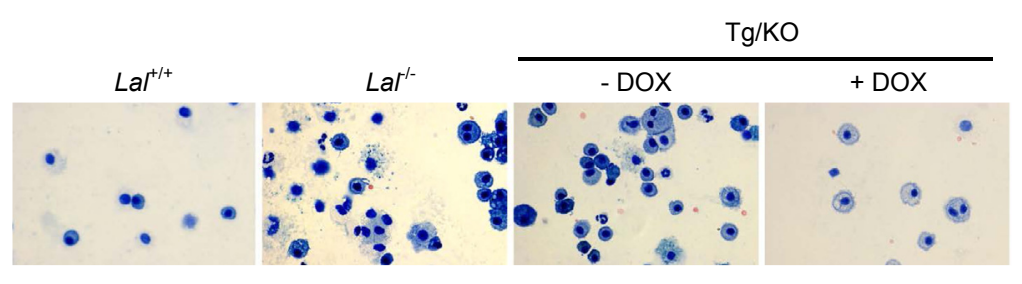

\section{C}
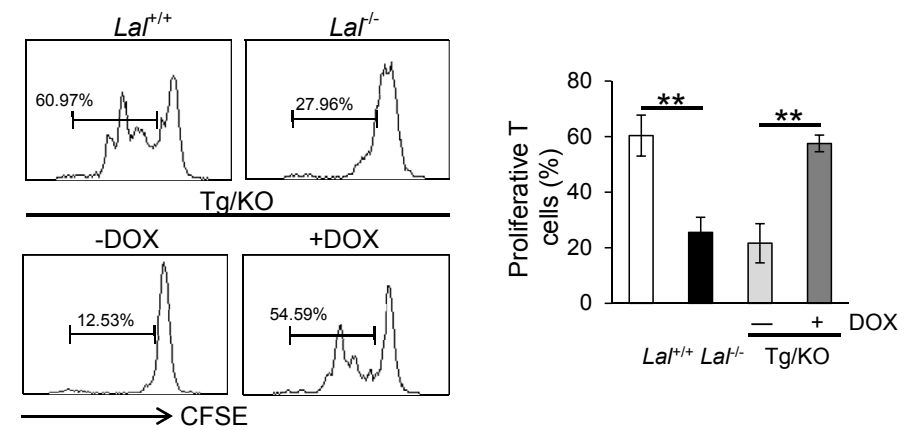

Figure 3 hLAL expression in lung epithelial cells decreases abnormal expansion of $\mathrm{Ly}_{6 \mathrm{G}}{ }^{+} \mathrm{CD}_{11} \mathrm{~b}^{+}$cells in $\mathrm{Lal}^{-/-}$mice. A: The percentages of Ly6G $\mathrm{G}^{+} \mathrm{CD} 11 \mathrm{~b}^{+}$ cells in the lung, blood, bone marrow (BM), and spleen of $\mathrm{Lal}^{+/+}, \mathrm{Lal}^{-/-}$, doxycycline-treated (+DOX), or untreated $(-\mathrm{DOX}) \mathrm{CCSP}-\mathrm{Tg} / \mathrm{KO}(\mathrm{Tg} / \mathrm{KO}) \mathrm{mice}$. B: Kwik-Diff staining of cells in the same amount of BALF from $\mathrm{Lal}^{+/+}, \mathrm{Lal}^{-/-}$, doxycycline-treated, or untreated CCSP-Tg/KO mice. C: Proliferation of car-

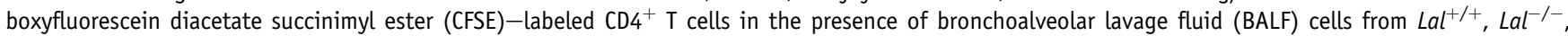
doxycycline-treated, or untreated CCSP-Tg/KO mice was analyzed by flow cytometry. The ratio between $\mathrm{CD}^{+}{ }^{+} \mathrm{T}$ cells and BALF cells was $5: 1$. Peaks represent cell division cycles. Data are expressed as means \pm SD (A and $\mathbf{C}) . n=4$ (A and $\mathbf{C}) .{ }^{*} P<0.05,{ }^{*} P<0.01$. Original magnification, $\times 400$ (B). MDSC, myeloidderived suppressor cell.

observation, mRNA syntheses of these cytokines and chemokines in the lungs were further investigated. mRNA levels of IL-6, CCL2, TNF- $\alpha$, CCL5, IL-10, and IL-1 $\beta$ were significantly down-regulated in the lungs of doxycyclinetreated CCSP-Tg/KO mice, whereas the GM-CSF level remained unchanged (Figure 5).

\section{hLAL Expression Reduces Tumor Cell Proliferation and Migration}

We further investigated whether down-regulation of cytokines and chemokines in BALF has an impact on tumor cell in vitro proliferation and migration. BALF supernatants from $\mathrm{Lal}^{+/+}, \mathrm{Lal}^{-1-}$, doxycycline-treated, or doxycyclineuntreated CCSP-Tg/KO mice were added into the culture medium of LLC cells for 72 hours. Compared with BALF from doxycycline-untreated $\mathrm{CCSP}-\mathrm{Tg} / \mathrm{KO}$ mice, BALF from doxycycline-treated $\mathrm{CCSP}-\mathrm{Tg} / \mathrm{KO}$ mice significantly decreased LLC cell proliferation in vitro (Figure 6A). The same observation was also made in B16 melanoma cells (Figure 6A).
Because cell migration contributes to metastasis, in vitro tumor cell migration assay was performed. LLC or B16 melanoma cells were treated with mitomycin $\mathrm{C}$ to eliminate the potential effects of cell proliferation in these assays. Fifteen hours after being cultured with $\mathrm{Lal}^{-1-}$ BALF, LLC or B16 melanoma cells migrated more efficiently into the area of an artificial wound area compared with those cells cultured with $\mathrm{Lal}^{+/+}$BALF (Figure 6B). However, delayed migration toward the scratch was observed in LLC or B16 melanoma cells cultured with BALF from doxycyclinetreated CCSP-Tg/KO mice. Taken together, these results suggest that lung epithelial hLAL expression reduced tumor cell proliferation and migration in vitro, which may explain why lung epithelial hLAL expression in $\mathrm{Lal}^{-/-}$mice reduced B16 melanoma cell metastasis in vivo (Figure 2).

\section{hLAL Expression Reduces Transendothelial Migration, EC Proliferation, and Migration}

Transendothelial migration of leukocytes is a critical step in the inflammatory response. ${ }^{23}$ In addition, transendothelial 

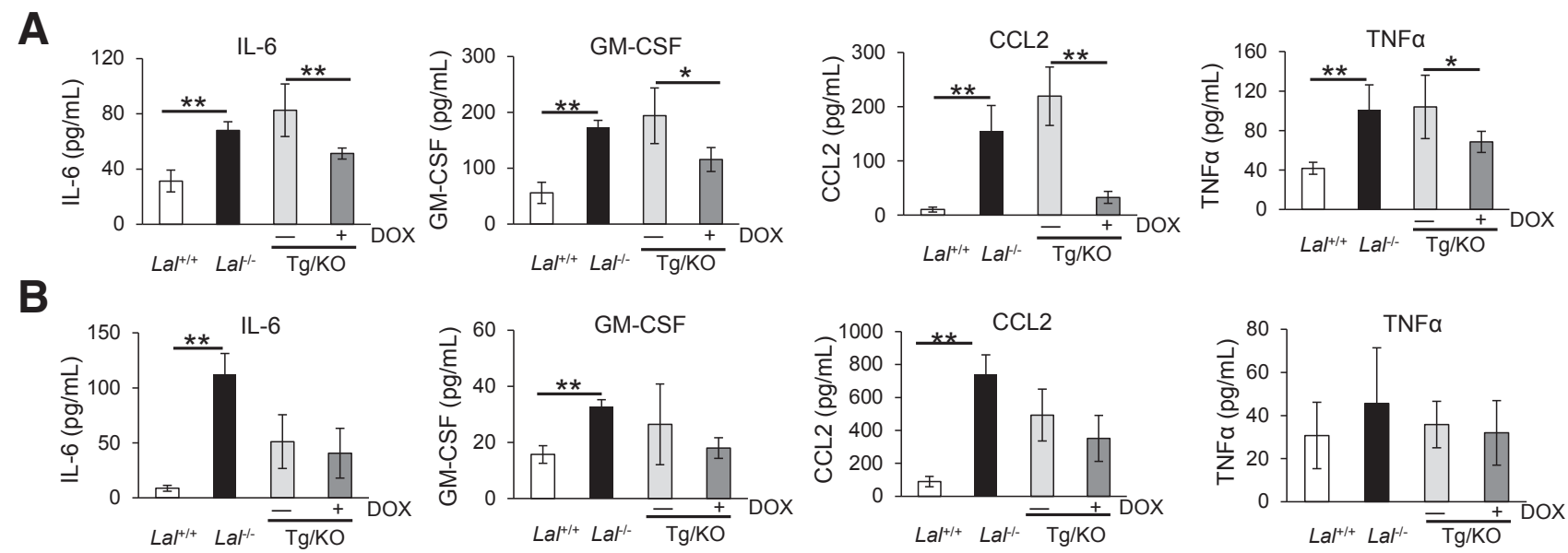

Figure 4 hLAL expression in lung epithelial cells reduces secretion of cytokines/chemokines in $\mathrm{Lal}^{-/-}$mice. The concentrations of IL-6, granulocytemacrophage colony-stimulating factor (GM-CSF), chemokine ligand (CCL) 2, and tumor necrosis factor (TNF)- $\alpha$ in the bronchoalveolar lavage fluid (A) and plasma (B) of $\mathrm{Lal}^{+/+}, \mathrm{Lal}^{-/-}$, doxycycline-treated (+DOX), or untreated (-DOX) CCSP-Tg/KO $(\mathrm{Tg} / \mathrm{KO})$ mice were determined by enzyme-linked immunosorbent assay. Data are expressed as means $\pm \mathrm{SD}(\mathbf{A}$ and $\mathbf{B}) . n=4$ to $5(\mathbf{A}$ and $\mathbf{B}) .{ }^{*} P<0.05,{ }^{* *} P<0.01$.

migration plays an important role in cancer metastasis. ${ }^{24} \mathrm{We}$ have recently reported that LAL deficiency increased MDSC and $\mathrm{T}$ cell transendothelial migration. ${ }^{18}$ The cytokines and chemokines in the BALF might induce the transendothelial migration of leukocytes. To mimic the in vivo condition, BALF was added into the culture medium of the lower chamber of transwell plates with bone marrow cells seeding on top of the primary lung EC monolayer in the upper chamber. Four hours later, the number of bone marrow cells that had migrated to the lower chamber was counted. There were significantly fewer bone marrow cells migrating to the lower chamber in which BALF from doxycycline-treated $\mathrm{CCSP}-\mathrm{Tg} / \mathrm{KO}$ mice was added than those migrating to the chamber added with untreated CCSP-Tg/KO BALF (Figure 7A). Similarly, the transmigrating ability of MDSCs or $\mathrm{T}$ cells was significantly reduced when BALF from doxycycline-treated CCSP-Tg/KO mice was added (Figure 7, B and C). To examine whether the reduced bone marrow cell transmigration was because of the decreased secretion of cytokines into BALF, the transwell study was further performed with BALF pretreated with anti-IL-6, anti-CCL2, or anti-TNF- $\alpha$ neutralizing antibodies. Results showed that bone marrow cell transmigration was significantly inhibited when doxycycline-untreated CCSP-Tg/KO BALF was treated with anti-IL-6, CCL2, or TNF- $\alpha$ antibodies (Figure 7A). Combination of these three neutralizing antibodies further blocked the transendothelial migration by doxycycline-untreated CCSP-Tg/KO BALF (Figure 7A). Therefore, secretion of lung epithelial LAL expressionreduced cytokines (especially IL-6) into the BALF is, at least in part, responsible for the decreased accumulation of myeloid cells in the BALF.
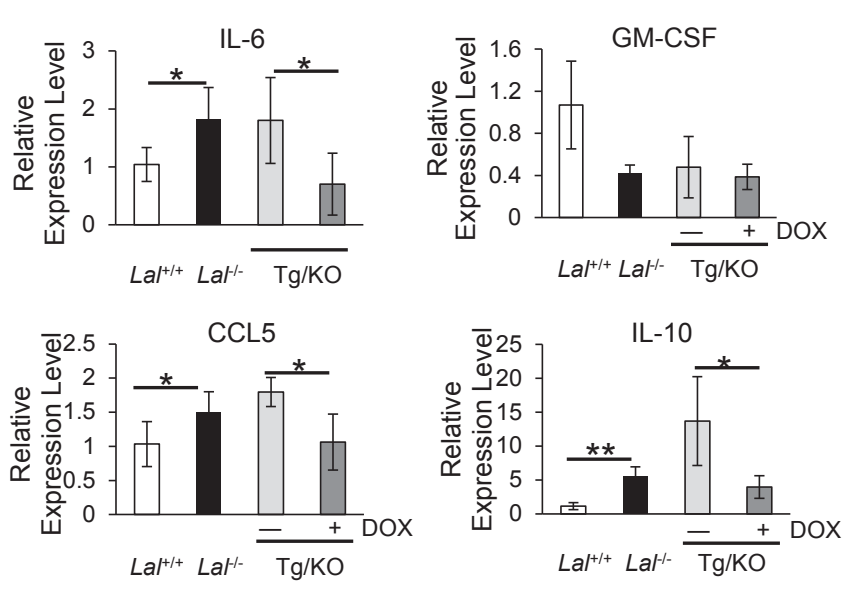
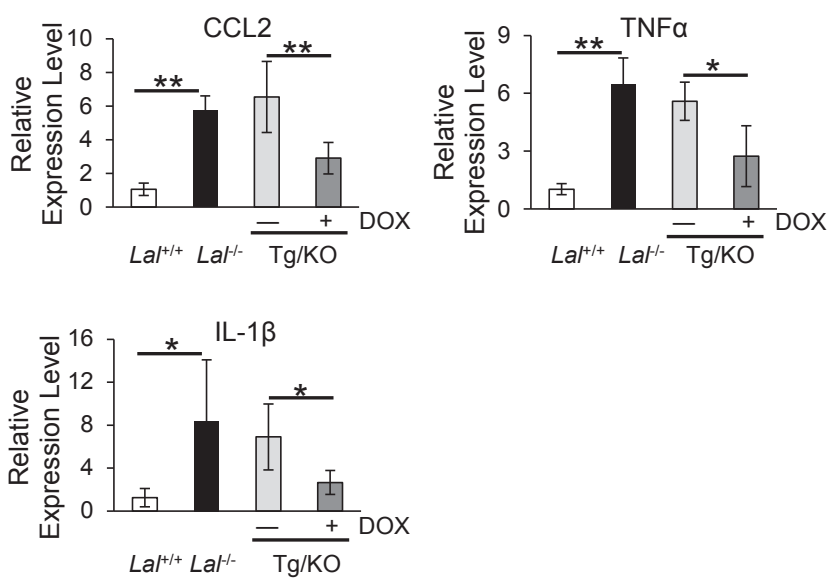

Figure 5 hLAL expression in lung epithelial cells reduces synthesis of cytokines/chemokines in $\mathrm{Lal}^{-/-}$mice. Real-time PCR analysis of mRNA expression levels of cytokines/chemokines in the lungs of $\mathrm{Lal}^{+/+}, \mathrm{Lal}^{-/-}$, doxycycline-treated (+DOX), or untreated (-DOX) CCSP- $\mathrm{Tg} / \mathrm{KO}(\mathrm{Tg} / \mathrm{KO}) \mathrm{mice}$. The relative gene expression was normalized to glyceraldehyde-3-phosphate dehydrogenase mRNA, and analysis was performed by the $2^{-\Delta \Delta C T}$ method. Data are expressed as means \pm SD. $n=4$. ${ }^{*} P<0.05,{ }^{*} P<0.01$. CCL, chemokine ligand; GM-CSF, granulocyte-macrophage colony-stimulating factor; TNF- $\alpha$, tumor necrosis factor- $\alpha$. 
A

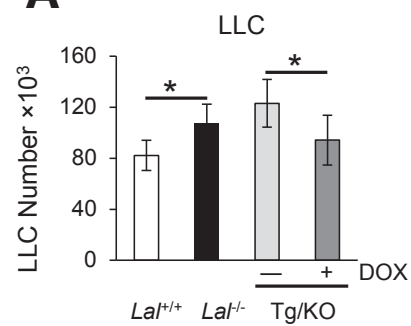

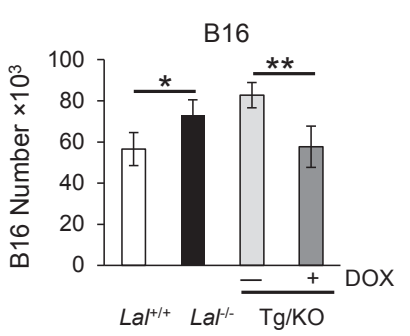

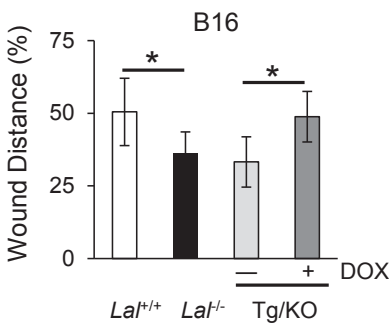

Figure 6 hLAL expression in lung epithelial cells reduces tumor cell proliferation and migration. A: Doxycycline (DOX)-treated CCSP-Tg/KO (Tg/KO) bronchoalveolar lavage fluid (BALF) decreases tumor cell proliferation in in vitro culture study. LLC or B16 melanoma cells $\left(5 \times 10^{3}\right)$ were cultured with BALF from $\mathrm{Lal}^{+/+}, \mathrm{Lal}^{-/-}$, and CCSP-Tg/KOmice in vitro for 72 hours, and the number of LLC or B16 melanoma cells were counted. B: Doxycycline-treated CCSP-Tg/K0 BALF decreased tumor cell migration. Tumor cell migration was assessed by the in vitro wound healing assay in the presence of mitomycin $C$ after cultured with BALF from $\mathrm{Lal}^{+/+}, \mathrm{Lal}^{-/-}$, and CCSP-Tg/KO mice. Data are expressed as means $\pm \mathrm{SD}(\mathbf{A}$ and $\mathbf{B}) . n=4(\mathbf{A}$ and $\mathbf{B}) .{ }^{*} P<0.05,{ }^{* *} P<0.01$.

The effects of CCSP-Tg/KO BALF on $\mathrm{Lal}^{+/+}$EC proliferation and migration were further investigated. When treated with $\mathrm{Lal}^{-1-}$ BALF, $\mathrm{Lal}^{+/+}$ECs showed increased proliferation compared with those treated with $\mathrm{Lal}^{+/+}$BALF. However, the increased proliferation of $\mathrm{Lal}^{+/+}$ECs was reversed by doxycycline-treated CCSP-Tg/KO BALF (Figure 7D). Furthermore, the in vitro wound healing assay showed increased migration (reduced wound distance) of $\mathrm{Lal}^{+/+}$ECs treated with $\mathrm{Lal}^{-/-}$BALF at 15 hours after generating the scratch, whereas the increased EC migration
A
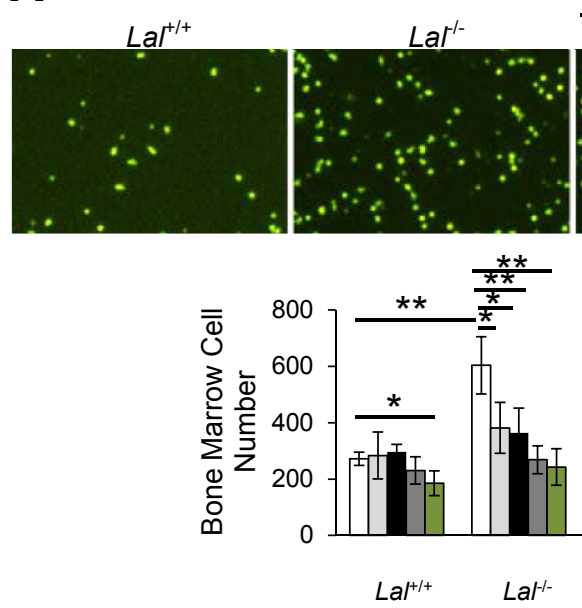

B

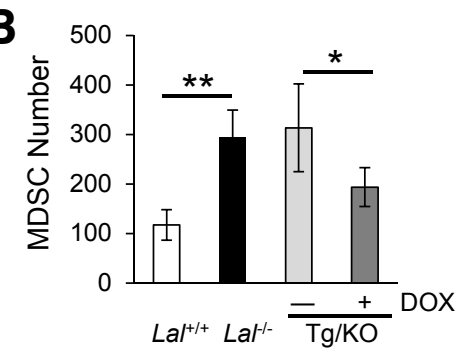

D

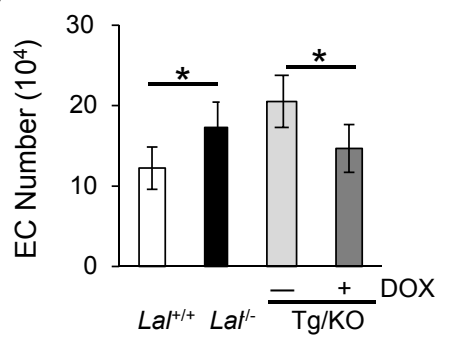

$\mathrm{Tg} / \mathrm{KO}$
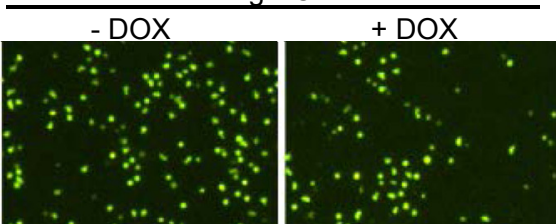

$\square C \lg G$

$\square$ Anti-CCL2

- Anti-TNFa

$\square$ Anti-IL-6

$\square$ Anti-all

DOX

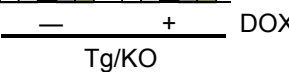

C

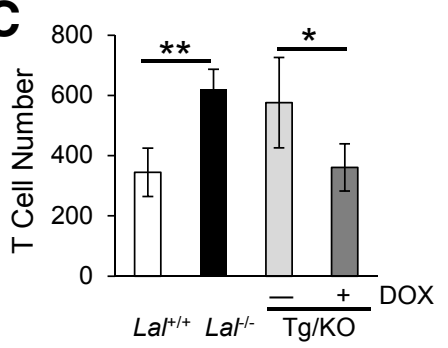

E

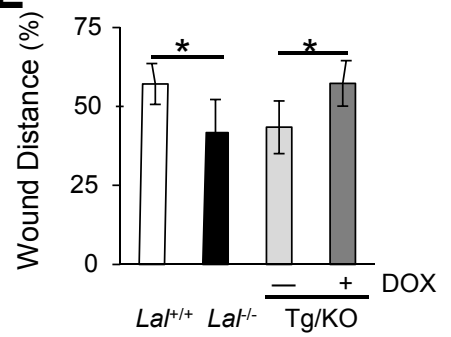

Figure 7 hLAL expression in lung epithelial cells reduces transendothelial migration, endothelial cell proliferation, and migration. A: Doxycycline (DOX)-treated CCSP-Tg/KO ( $\mathrm{Tg} / \mathrm{KO})$ bronchoalveolar lavage fluid (BALF) decreases bone marrow cell transendothelial migration by transwell assay. Neutralizing antibodies against IL-6, chemokine ligand (CCL) 2, or tumor necrosis factor (TNF)- $\alpha$ were individually or in combination added to the BALF before seeding bone marrow cells. $\mathbf{B}$ : Doxycycline-treated CCSP-Tg/KO BALF decreases myeloid-derived suppressor cell (MDSC) transendothelial migration by transwell assay. C: Doxycycline-treated CCSP-Tg/KO BALF decreases $\mathrm{CD}^{+} \mathrm{T}$ cell transendothelial migration. D: Doxycyclinetreated CCSP-Tg/KO BALF decreases endothelial cell $(E C)$ proliferation. ECs were co-cultured with BALF from $\mathrm{Lal}^{+/+}, \mathrm{Lal}^{-/-}, \mathrm{CCSP}-\mathrm{Tg} / \mathrm{KO}$ mice in vitro for 72 hours, and the number of ECs was counted. E: Doxycycline-treated CCSP-Tg/KO BALF decreases EC migration by the in vitro wound healing assay in the presence of mitomycin C. Data are expressed as means $\pm \mathrm{SD}(\mathbf{A}-\mathbf{E}) . n=4(\mathbf{A}-\mathbf{E}) .{ }^{*} P<0.05$, $* * P<0.01$. Original magnification, $\times 40$. 
was reduced when doxycycline-treated CCSP-Tg/KO BALF was added (Figure 7E). Taken together, lung epithelial LAL expression in $\mathrm{Lal}^{-1-}$ mice decreased the stimulation of BALF on lung EC proliferation and migration.

\section{Discussion}

We have demonstrated that lipid metabolism controlled by LAL is critical for AT II epithelial cell homeostasis and alveolar genesis. ${ }^{13}$ Blockage of the LAL metabolic pathway in AT II epithelial cells results in exuberant inflammation, hyperplasia, and emphysema in the lung, which are caused by aberrant inflammatory gene expression in an agedependent manner. ${ }^{6}$ To further identify the roles of LAL in AT II epithelial cells in tumorigenicity and metastasis, hLAL was specifically reintroduced into the $\mathrm{Lal}^{-1-}$ mice and its expression was driven by AT II epithelial cellspecific promoter. AT II epithelial cell-specific hLAL expression in CCSP-Tg/KO mice was verified at the protein expression level (Figure 1A). Histological analysis revealed that the hLAL expression in lung AT II epithelial cells reversed alveolar destruction in doxycycline-treated CCSP$\mathrm{Tg} / \mathrm{KO}$ mice (Figure 1, B and C). Therefore, LAL in AT II epithelial cells is essential for maintaining normal alveolar structure and functions in the lung.

LAL deficiency has been found to facilitate melanoma growth and metastasis in the lung. ${ }^{11}$ The functional role of AT II epithelial cells in this pathogenic process is not clear. Herein, AT II epithelial cell-specific expression of hLAL in CCSP-Tg/KO mice reduced $\mathrm{B} 16$ melanoma cell metastasis in the lung (Figure 2), accompanied by a decrease in the number of tumor-promoting MDSCs (Figure 3). The reduced MDSC level was also observed in the peripheral blood of doxycycline-treated CCSP-Tg/KO mice (Figure 3). MDSCs that possess immunosuppression on $\mathrm{T}$ cells are a critical component in the tumor microenvironment. Indeed, BALF cells (mainly MDSCs) from AT II epithelial cell-specific expression of hLAL reduced T cell suppression (Figure 3C). Consistent with this observation, we have shown that MDSCs from $\mathrm{Lal}^{-1-}$ mice directly stimulate proliferation of B16 melanoma cells in vitro, as well as growth and metastasis in vivo. ${ }^{11}$ Myeloid-specific expression of hLAL in $\mathrm{Lal}^{-1-}$ mice (the c-fms- $\mathrm{Tg} / \mathrm{KO}$ mouse model) reduced the systemic MDSC influx and inflammation-associated pathogenesis in multiple organs, ${ }^{8}$ including tumor growth and metastasis. ${ }^{11}$ This is mainly by correction of MDSC expansion starting from the early developing stage of granular myeloid progenitor cells in the bone marrow. ${ }^{25}$ Myeloid-specific expression of hLAL in $\mathrm{Lal}^{-1-}$ mice reduced MDSCs' ability to stimulate cancer cell proliferation and overcome immune rejection of cancer metastasis, which switches metabolic reprogramming of MDSCs. ${ }^{26}$

In a separate LAP-Tg/KO mouse model, in which hepatocyte-specific expression of hLAL was driven by the hepatocyte cell-specific LAP promoter, LAL expression reduced MDSC expansion, increased T cell population, and reduced tumor metastasis through down-regulation of inflammatory cytokines and chemokines in the liver. ${ }^{17}$ Similarly, the concentrations of these cytokines (ie, IL-6, TNF- $\alpha$, GM-CSF, and CCL2) were increased in the BALF of the $\mathrm{Lal}^{-1-}$ lung, which were reduced in the BALF of the doxycycline-treated CCSP-Tg/KO mice (Figure 4). This observation was confirmed by mRNA expression levels (Figure 5). More important, BALF from $\mathrm{Lal}^{-1-}$ mice facilitated LLC and B16 cell proliferation and migration in vitro, which was corrected by AT II epithelial cellspecific expression of hLAL in CCSP-Tg/KO mice (Figure 6). Therefore, LAL production in various cells (eg, AT II epithelial cells, hepatocytes, and myeloid cells) is a safe guard to block cancer cell progression and metastasis via controlling MDSCs and tumor-promoting cytokines and chemokines in various organs.

As we reported previously, the underlying mechanisms by which LAL deficiency causes infiltration of MDSCs in the lung also depend on ECs, which constitute barriers regulating leukocyte transmigration to the site of inflammation. ${ }^{18} \mathrm{Lal}^{-1-}$ MDSCs promoted in vivo angiogenesis (including in tumor model), lung EC tube formation, and proliferation. ${ }^{18}$ In the present study, $\mathrm{Lal}^{-1-}$ BALF significantly increased lung EC proliferation, migration, and permeability of bone marrow cells, all of which were reversed by BALF from the doxycycline-treated CCSP-Tg/ KO mice (Figure 7). Addition of neutralizing antibodies against IL-6, TNF- $\alpha$, and CCL2 into the untreated CCSP$\mathrm{Tg} / \mathrm{KO} \mathrm{BALF}$ inhibited transmigration of bone marrow cells (Figure 7), further supporting that proinflammatory cytokines in BALF play pleiotropic roles in mediating LAL functions, which suggested that epithelial hLAL expression-reduced cytokines/chemokines affect the ability of leukocytes to transmigrate.

Taken together, AT II epithelial cells play a central role in the lung to control MDSC homeostasis, EC permeability, and tumorigenesis. The metabolic pathway controlled by LAL in AT II epithelial cells tightly regulates synthesis and secretion of proinflammatory cytokines and chemokines, and immune cells that actively participate in the regional pulmonary microenvironment to form niches for tumor growth and metastasis in the lung. Therefore, LAL may be used in immunotherapy to treat various inflammationinduced cancer forms.

\section{References}

1. Yan $\mathrm{C}, \mathrm{Du} \mathrm{H}$ : Alveolus formation: what have we learned from genetic studies? J Appl Physiol 2004, 97:1543-1548

2. Li Y, Du H, Qin Y, Roberts J, Cummings OW, Yan C: Activation of the signal transducers and activators of the transcription 3 pathway in alveolar epithelial cells induces inflammation and adenocarcinomas in mouse lung. Cancer Res 2007, 67:8494-8503

3. Li Y, Qu P, Wu L, Li B, Du H, Yan C: Api6/AIM/Spalpha/CD5L overexpression in alveolar type II epithelial cells induces spontaneous lung adenocarcinoma. Cancer Res 2011, 71:5488-5499 
4. Qu P, Du H, Wang X, Yan C: Matrix metalloproteinase 12 overexpression in lung epithelial cells plays a key role in emphysema to lung bronchioalveolar adenocarcinoma transition. Cancer Res 2009, 69:7252-7261

5. Wu L, Du H, Li Y, Qu P, Yan C: Signal transducer and activator of transcription 3 (Stat3C) promotes myeloid-derived suppressor cell expansion and immune suppression during lung tumorigenesis. Am J Pathol 2011, 179:2131-2141

6. Lian X, Yan C, Yang L, Xu Y, Du H: Lysosomal acid lipase deficiency causes respiratory inflammation and destruction in the lung. Am J Physiol Lung Cell Mol Physiol 2004, 286:L801-L807

7. Qu P, Shelley WC, Yoder MC, Wu L, Du H, Yan C: Critical roles of lysosomal acid lipase in myelopoiesis. Am J Pathol 2010, 176: 2394-2404

8. Yan C, Lian X, Li Y, Dai Y, White A, Qin Y, Li H, Hume DA, Du H: Macrophage-specific expression of human lysosomal acid lipase corrects inflammation and pathogenic phenotypes in lal-/- mice. Am J Pathol 2006, 169:916-926

9. Ding X, Du H, Yoder MC, Yan C: Critical role of the mTOR pathway in development and function of myeloid-derived suppressor cells in lal/- mice. Am J Pathol 2014, 184:397-408

10. Lian X, Yan C, Qin Y, Knox L, Li T, Du H: Neutral lipids and peroxisome proliferator-activated receptor-\{gamma\} control pulmonary gene expression and inflammation-triggered pathogenesis in lysosomal acid lipase knockout mice. Am J Pathol 2005, 167:813-821

11. Zhao T, Du H, Ding X, Walls K, Yan C: Activation of mTOR pathway in myeloid-derived suppressor cells stimulates cancer cell proliferation and metastasis in lal-/- mice. Oncogene 2015, 34:1938-1948

12. Tichelaar JW, Lu W, Whitsett JA: Conditional expression of fibroblast growth factor-7 in the developing and mature lung. J Biol Chem 2000 275:11858-11864

13. Li Y, Qin Y, Li H, Wu R, Yan C, Du H: Lysosomal acid lipase overexpression disrupts lamellar body genesis and alveolar structure in the lung. Int J Exp Pathol 2007, 88:427-436

14. Qu P, Du H, Li Y, Yan C: Myeloid-specific expression of Api6/AIM/Sp alpha induces systemic inflammation and adenocarcinoma in the lung. J Immunol 2009, 182:1648-1659
15. Qu P, Roberts J, Li Y, Albrecht M, Cummings OW, Eble JN, Du H, Yan C: Stat3 downstream genes serve as biomarkers in human lung carcinomas and chronic obstructive pulmonary disease. Lung Cancer 2009, 63:341-347

16. Wu L, Yan C, Czader M, Foreman O, Blum JS, Kapur R, Du H: Inhibition of PPARgamma in myeloid-lineage cells induces systemic inflammation, immunosuppression, and tumorigenesis. Blood 2012, 119:115-126

17. Du H, Zhao T, Ding X, Yan C: Hepatocyte-specific expression of human lysosome acid lipase corrects liver inflammation and tumor metastasis in lal-/- mice. Am J Pathol 2015, 185: 2379-2389

18. Zhao T, Ding X, Du H, Yan C: Myeloid-derived suppressor cells are involved in lysosomal acid lipase deficiency-induced endothelial cell dysfunctions. J Immunol 2014, 193:1942-1953

19. Liang CC, Park AY, Guan JL: In vitro scratch assay: a convenient and inexpensive method for analysis of cell migration in vitro. Nat Protoc 2007, 2:329-333

20. Balkwill F: Cancer and the chemokine network. Nat Rev Cancer 2004, 4:540-550

21. Talmadge JE, Gabrilovich DI: History of myeloid-derived suppressor cells. Nat Rev Cancer 2013, 13:739-752

22. Burkholder B, Huang RY, Burgess R, Luo S, Jones VS, Zhang W, Lv ZQ, Gao CY, Wang BL, Zhang YM, Huang RP: Tumor-induced perturbations of cytokines and immune cell networks. Biochim Biophys Acta 2014, 1845:182-201

23. Muller WA: Mechanisms of transendothelial migration of leukocytes. Circ Res 2009, 105:223-230

24. Reymond N, d'Agua BB, Ridley AJ: Crossing the endothelial barrier during metastasis. Nat Rev Cancer 2013, 13:858-870

25. Qu P, Yan C, Blum JS, Kapur R, Du H: Myeloid-specific expression of human lysosomal acid lipase corrects malformation and malfunction of myeloid-derived suppressor cells in lal-/- mice. J Immunol 2011, 187 3854-3866

26. Ding X, Wu L, Yan C, Du H: Establishment of lal-/- myeloid lineage cell line that resembles myeloid-derived suppressive cells. PLoS One 2015, 10:e0121001 